\title{
Hypopomus Gill: nova apomorfia e notas sobre suas relações filogenéticas (Teleostei, Gymnotiformes, Rhamphichthyoidea)
}

\author{
Mauro L. Triques \\ Departamento de Zoologia, Instituto de Ciências Biológicas, Universidade Federal de Minas Gerais. Caixa Postal 486, \\ 31270-901 Belo Horizonte, Minas Gerais, Brasil. E-mail: triques@mono.icb.ufmg.br
}

\begin{abstract}
Hypopomus Gill: new apomorphy and notes on its phylogenetic relationships (Teleostei, Gymnotiformes, Rhamphichthyoidea). The monophyly of Hypopomus Gill, 1864 is corroborated, on the basis of the presence of mouth corner upturned, homoplastically also in Steatogenys Boulenger, 1898. Its synonymy with Parupygus Hoedeman, 1962 is corroborated. Three of seven synapomorphies attributed to the monophyly of Hypopomidae are refuted (posterior region of parasphenoid narrow, depression on the dorsal surface of bashyal and trapezoidal opercle) and four other are presented to unite Hypopomus to Rhamphichthyidae (lower jaw included; anterior elongation of dentary; elongation of palatine cartilage and ascending process of mesopterygoid backward curved). Thus, further studies are necessary to assess the phylogenetic position of the genus.
\end{abstract}

KEY WORDS. Cladistics; Ostariophysi; neotropical region; Parupygus; Steatogenys.

RESUMO. O monofiletismo de Hypopomus Gill, 1864 é corroborado pela presença de canto da boca curvo para cima, homoplasticamente também em Steatogenys Boulenger, 1898. Sua sinonímia com Parupygus Hoedeman, 1962 é corroborada. Três de sete apomorfias previamente atribuídas para definir o monofiletismo de Hypopomidae são refutadas (região posterior do paresfenóide estreita, depressão na face dorsal do basi-hial e opérculo trapezoidal) e outras quatro são propostas para unir Hypopomus a Rhamphichthyidae (mandíbula incluída; alongamento anterior do dentário; cartilagem palatina alongada e processo ascendente do mesopterigóide curvo para trás). Assim, estudos mais elaborados são necessários para avaliar a posição filogenética do gênero.

PALAVRAS-CHAVE. Cladística; Ostariophysi; Parupygus; região neotropical; Steatogenys.

Hypopomus foi proposto por Gill (1864: 152) para incluir Rhamphichthys mulleri Kaup, 1856, sem apresentar uma diagnose; outra espécie similar, $R$. artedi Kaup, 1856, não foi mencionada. Parupygus foi proposto por Hoedeman (1962: 58), considerado como intimamente relacionado às espécies de Hypopomus e separado deste pela forma da cabeça, posição dos olhos e da narina posterior; outras características apontadas por ele, mas de abrangência ou de utilidade diagnóstica não esclarecidas, são a fontanela parietal muito estreita e longa, bem como focinho similar ao de Sternopygus Müller \& Troschel, 1848 (gênero de Sternopygidae). Mago-Leccia (1978: 14) propôs Hypopomidae, para incluir os gêneros Hypopomus, Hypopygus Hoedeman, 1962, Steatogenys Boulenger, 1898 e Parupygus; Hypopomidae foi incluída em Rhamphichthyoidea, juntamente com Rhamphichthyidae. Mago-Leccia (1994: 47) sinonimizou Parupygus a Hypopomus, sem justificativas. Albert (2001) seguiu Mago-Leccia (1994) neste sentido, e apresentou uma sinapomorfia para o gênero: lâmina óssea descendente, do osso maxilar, com margem ventral angulosa (sua apomorfia número 37) e definiu Hypopomidae com base em sete apomorfias: (1) componente dérmico do vômer composto não ossificado; (2) por- ção posterior do paresfenóide estreitada, ocupando menos que a metade da superfície ventral do neurocrânio; (3) um ramo do nervo da linha lateral posterior passando junto do ramo recorrente do nervo ântero-ventral da linha lateral; (4) osso opercular de formato trapezoidal; (5) superfície dorsal do basi-hial côncavo; (6) processo ântero-ventral do coracóide de tamanho reduzido; e (7) segundo espinho hemal dorsal (DHS na sigla em inglês) curvo. Albert (2003: 495) considerou válida apenas $H$. artedi, neste gênero.

No entanto, o exame de material diafanizado de diversas espécies de hipopomídeos e ranfictiídeos mostrou que alguns caracteres não são apomorfias de Hypopomidae ao mesmo tempo em que outras apomorfias, novas, unem Hypopomus a Rhamphichthyidae. Uma nova apomorfia, de anatomia externa, foi encontrada para Hypopomus e um estudo detalhado corroborou a sinonímia entre Hypopomus e Parupygus. Os exemplares estudados estão listados em Triques (1996b, 1997, 1998, 1999, 2005a) e Triques \& Khamis (2003). A análise cladística (HenNig 1966, Wiley 1981, entre muitos outros) aqui elaborada utilizou-se do método do grupo externo, proposto por Watrous \& WheEler (1981) e desenvolvido por MADDison et al. (1984) e Nixon \& CARPenter 
(1993) para a polarização dos caracteres. Diafanizações seguiram o método de TAYLOR \& VAN DYKE (1985); dissecções seguiram Weitzman (1974) e a nomenclatura esquelética utilizada foi a de Castro \& Castro (1987). Proporções corporais e outros caracteres de anatomia externa e interna foram estudados e estão caracterizados em Triques (1996a, 2005a: 37-38); além destes, foram contados os raios das nadadeiras anal e peitoral, bem como as séries de escamas acima da linha lateral, no nível da maior altura do corpo; o tamanho e formato das escamas dorsais, da linha lateral e ventrais no corpo foram também avaliados. Para efeito de comparação entre os gêneros nominais Hypopomus e Parupygus, foram utilizados dados de material tipo das espécies e, em alguns casos, de exemplares identificados com base na comparação com este material.

\section{Hypopomus Gill, 1864}

Uma nova apomorfia, de anatomia externa, foi encontrada para o gênero: canto da boca curvo para cima, lembrando o formato de um gancho (Fig. 1). De todos os Gymnotiformes conhecidos, apenas Hypopomus e Steatogenys possuem o canto da boca curvo para cima (não estudado em $S$. ocellatus Crampton, Thorsen \& Albert, 2004). Hypopomus é basal em Hypopomidae e Steatogenys é derivado (Albert 2001) nesta família, de modo que, por uma questão de parcimônia global dos caracteres, considerei esta estrutura como um caráter homoplástico de Steatogenys e de Hypopomus.

Todas as características apresentadas por HoEdeman (1962) para separar as espécies nominais de Parupygus das de Hypopomus foram estudas, bem como a altura do olho em relação à altura cefálica, avaliada como a distância entre a margem ocular inferior e a linha mediana ventral cefálica, em relação à maior altura da cabeça; o comprimento do focinho em relação ao comprimento cefálico; a distância entre a narina posterior e a margem anterior do olho, com relação à distância pós-ocular; a distância entre a narina posterior e a margem ocular anterior, em relação ao comprimento cefálico; as larguras cefálica (no nível do opérculo) e corporal, com relação à distância da extremidade anterior do focinho até a base do último raio da nadadeira anal; posição da narina anterior em relação ao lábio superior; a distância da extremidade anterior do focinho até a narina posterior, em relação ao comprimento do focinho e, finalmente, a escamação corporal quanto à distribuição das escamas e quanto ao formato destas. Nenhuma diferença foi encontrada entres estes gêneros nominais nem entre as espécies nominais. Uma vez que o material tipo das espécies nominais de Hypopomus e Parupygus apresenta canto da boca curvo para cima, fica claro que Parupygus é sinônimo júnior de Hypopomus e que este é um grupo monofilético, corroborando Mago-Leccia (1994) e Albert (2001: 68).

\section{Relações filogenéticas de Hypopomus}

Algumas sinapomorfias indicam que Hypopomus é mais intimamente relacionado a Rhamphichthyidae do que aos demais representantes de Hypopomidae, apresentadas subseqüentemente.

Revista Brasileira de Zoologia 24 (3): 717-720, setembro 2007

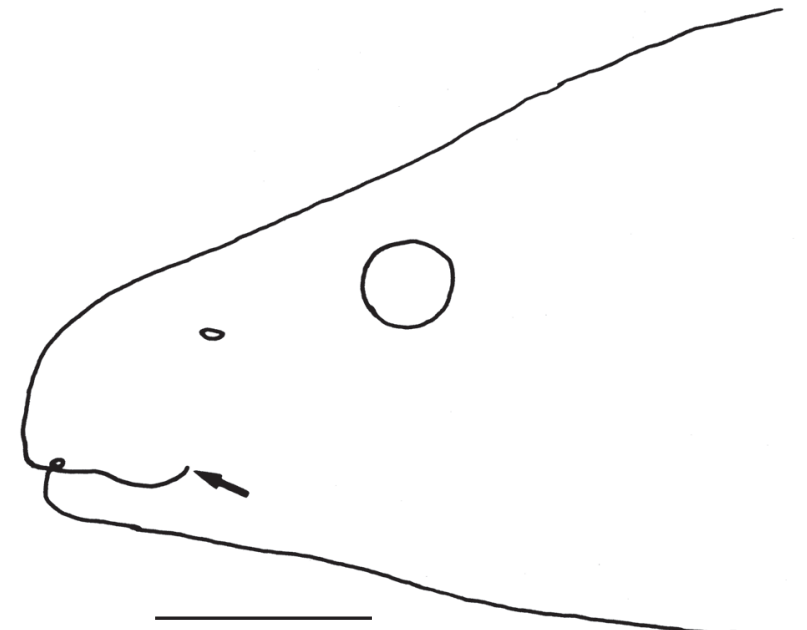

Figura 1. Perfil da região anterior da cabeça de Hypopomus artedi, ZMA 102.375 (holótipo de Parupygus savannensis); comprimento cefálico 21,0 mm; barra indica $5 \mathrm{~mm}$. Seta indica o canto da boca.

Somente em Rhamphichthyidae, Hypopomus e Brachyhypopomus occidentalis (Regan, 1914) ocorre uma mandíbula completamente incluída na maxila superior, de modo que a boca abre-se ventralmente (MAGo-Leccia 1994: 169, fig. 69a). Em nenhum outro grupo de Gymnotiformes ocorre a inclusão completa da mandíbula. Esta apomorfia indica que Hypopomus e $B$. occidentalis seriam parte de Rhamphichthyidae. As três próximas apomorfias indicam que apenas Hypopomus seria parte desta família.

Somente em Hypopomus (apenas um exemplar de H. artedi analisado) e Rhamphichthyidae (não visto em Iracema Triques, 1996), o dentário é alongado anteriormente e a cartilagem palatina é alongada e estendida para frente, unindo-se ao antorbital, constituindo-se em duas apomorfias deste grupo. Em Sternopygidae, esta cartilagem está ligada ao maxilar, e o dentário não se alonga anteriormente (Mago-Leccia 1978, figs 8 e 25; Triques 1993, fig. 11). Nos gêneros mais basais de Apteronotidae (Sternarchorhamphus Eigenmann, 1905 e Orthosternarchus Ellis, 1913), o alongamento do focinho ocorre de outras formas (TRIQUES 2005b: 145). Em Gymnotidae, Electrophoridae e Brachyhypopominae (Triques 1993, figs 5-7 e 10), o alongamento anterior do dentário e o alongamento da cartilagem palatina, ligada ao antorbital não se verifica. Fica claro, portanto, que o alongamento do focinho em Hypopomus + Rhamphichthyidae ocorreu através do alongamento de elementos esqueléticos diferentes daqueles envolvidos no alongamento do focinho de Apteronotidae e Sternopygidae. Assim, o alongamento do focinho em Apteronotidae, Sternopygidae e Hypopomus + Rhamphichthyidae ocorreu independentemente entre si, contando-se três passos evolutivos distintos.

Em todos os Gymnotiformes, o processo ascendente do mesopterigóide dirige-se para cima, exceto em Hypopomus e 
Rhamphichthys Müller \& Troschel, 1848 (respectivamente Chardon \& de la Hoz 1974: 18, fig. 2; Mago-Leccia 1994), onde ele é curvo para trás. Este caráter foi estudado em $H$. artedi (um exemplar diafanizado) e H. savannensis Hoedeman, 1962 (ChARDon \& DE LA Hoz 1974: 18, fig. 2). Este processo não ocorre em Gymnorhamphichthys Ellis, 1912 nem em Electrophorus Gill, 1864 e é de formato variável em Gymnotus Linnaeus, 1758.

Estas quatro apomorfias indicam que Hypopomus pertence a Rhamphichthyidae, mas Albert (2001: 68) sustenta o monofiletismo de Hypopomidae, incluindo Hypopomus, com base em sete apomorfias: (1) parte dérmica do vômer - igual à "parte laminar do vômer" em TRIQUEs (1993: 96), caráter 10 - não ossificado; (2) região posterior do paresfenóide estreito; (3) um ramo do nervo posterior do sistema látero-sensorial passando junto a um ramo recorrente do nervo ântero-ventral do sistema láterosensorial; (4) opérculo trapezoidal; (5) superfície dorsal do basihial côncava; (6) processo ântero-ventral do coracóide reduzido (exceto Steatogenys); (7) segundo espinho hemal dorsal (DHS, sigla em inglês) curvo. Algumas destas apomorfias podem ser questionadas, no entanto. Eu considero muito variável o formato do opérculo; assim, em Hypopygus lepturus Hoedeman, 1962, pode ser claramente trapezoidal ou quase triangular (Triques 1993, fig. 5), triangular em Brachyhypopomus beebei (Schultz, 1944), B. occidentalis, H. savannensis (CHARDON \& DE LA Hoz 1974: 18, fig. 2), Microsternarchus bilineatus Fernàndez Yépez, 1968 e Racenisia fibriipina Mago-Leccia, 1994 (MAGo-Leccia 1994: 176, fig. 77), trapezoidal em Steatogenys elegans (Steindachner, 1880) e Brachyhypopomus brevirostris (Steindachner, 1868) (obs. pess.), de modo que não considero este caráter uma sinapomorfia de Hypopomidae. A superfície dorsal côncava do basi-hial, caráter 162 de Albert (2001), é o caráter 30 de Triques (1993) de acordo com Albert (2001: 39) e que, na verdade, se trata de uma "depressão na face dorsal do base-hial" (TRIQues 1993: 107, 109; fig. 13), que ocorre também em Rhamphichthys e que, portanto, é um caráter ambíguo, uma vez que pode ser apomorfia de Rhamphichthyoidea, com uma reversão em Gymnorhamphichthys. A região posterior do paresfenóide é tão estreita em S. elegans e B. brevirostris quanto em Gymnorhamphichthys rondoni (Miranda Ribeiro, 1920) e Rhamphichthys sp. (observado num juvenil de 52,5 mm de comprimento padrão e 10,0 mm de comprimento cefálico, $R$. rostratus de Triques 1993: 91), de modo que não considero este caráter uma apomorfia de Hypopomidae. Assim, considero válidas quatro apomorfias para Hypopomidae, como definidas por AlbERT (2001).

As apomorfias que incluem Hypopomus em Rhamphichthyidae poderiam ser interpretadas como apomorfias de Rhamphichthyoidea, com reversão em Brachyhypopominae (Albert 2001). Esta hipótese, entretanto, duplica o número de passos num cladograma. Portanto, considerando-se válidas quatro apomorfias para unir Hypopomus com os demais Hypopomidae e quatro apomorfias para trazer Hypopomus para Rhamphichthyidae, uma análise cladística mais abrangente e profunda é necessária para avaliar o posicionamento filogenético de Hypopomus dentro de Rhamphichthyioidea.

\section{AGRADECIMENTOS}

A Frederico M. de Queiroz pela preparação do arquivo PDF; à Cláudia M. Jacobi pelo auxílio com o abstract (erros remanescentes são meus); a H. Nijssen, I. J. H. Isbrücker e J-C Hureau pelo empréstimo de material tipo; a Francisco Langeani Neto e John Sullivan pelo traslado de material tipo; a Naércio A. Menezes pela orientação durante meu doutorado; a Ricardo Campos-daPaz pelo auxílio com a literatura; a John G. Lundberg pela oportunidade na Amazônia e no INPA; a Heraldo A. Britski e José L. de Figueiredo pela permissão do uso de instalações da seção de peixes do Museu de Zoologia da Universidade de São Paulo; a Fernando A. da Siveira, pela discussão sobre o posicionamento de Hypopomus em Rhamphichthyoidea; à FAPESP, pela bolsa de doutoramento (proc. 91/1389-8); à FAPEMIG, pelo equipamento óptico concedido (proc. CRA 2146/97).

\section{REFERÊNCIAS BIBLIOGRÁFICAS}

Albert, J.S. 2001. Species diversity and phylogenetic systematics of American Knifefishes (Gymnotiformes, Teleostei). Miscellaneous Publications Museum of Zoology, University of Michigan (190): VI+127.

Albert, J.S. 2003. Family Apteronotidae, p. 497-502. In: R.E. Reis; S.O. Kullander \& C.J. Ferraris JR (Eds). Check List of the Freshwater Fishes of South and Central America. Porto Alegre, EDIPUCRS, XII+729p.

Castro, R.M.C. \& M.M.C. Castro. 1987. Proposta de uma nomenclatura osteológica para Characiformes (Pisces: Ostariophysi). Boletim do Museu Paraense "Emílio Goeldi", Série Zoologia, 3 (1): 25-32.

Chardon, M. \& E. DE LA Hoz. 1974. Towards an improved classification of the gymnotid fishes by the use of the splanchnochranium characters. Acta Biologica Iugoslavica 6 (1): 15-25.

GiLl, T. 1864. [Several points in ichthyology and conchology.]. Proceeding of the Academy of Natural Sciences of Philadelphia 16: 151-152

Hennig, W. 1966. Phylogenetic Systematics. Urbana, University of Illinois Press, 263p.

Hoedeman, J.J. 1962. Notes on the ichthyology of Surinam and other Guianas. 9 New records of Gymnotid fishes. Bulletin of Aquatic Biology 3 (26): 53-60.

Maddison, W.P.; M.J. Donoghue \&. R. Maddison. 1984. Outgroup analysis and parsimony. Systematic Zoology 33: 83-183.

Mago-Leccia, F. 1978. Los peces de la família Sternopygidae de Venezuela. Acta científica venezolana 29 (l): 1-89.

Mago-Leccia, F. 1994. Electric fishes of the continental waters of America. Caracas, Fundacion para el Desarrollo de las Ciencias Fisicas, Matematicas y Naturales, 223p.

Nixon, K.C. \& J.M. Carpenter. 1993. On outgroups. Cladistics 9: 413-426.

TAYlor, W.R. \& G.C. van DyKe. 1985. Revised procedures for staining and clearing small fishes and other vertebrates for 
bone and cartilage study. Cybium 9 (2): 107-119.

Triques, M.L. 1993. Filogenia dos gêneros de Gymnotiformes (Actinopterygii, Ostariophysi), com base em caracteres esqueléticos. Comunicações do Museu de Ciências e Tecnologia da PUCRS, Série Zoologia, 6: 85-130.

Triques, M.L. 1996a. Eigenmannia vicentespelaea, a new species of cave dweling electrogenic neotropical fish (Gymnotiformes, Sternopygidae). Révue Française d'Aquariologie et Herpetologie 23 (3/4): 1-4.

TRIQUes, M.L. 1996b. Iracema cayana, new genus and species of electrogenic neotropical freshwater fish (Rhamphichthyidae: Gymnotiformes: Ostariophysy: Actinopterygii). Révue Française d'Aquariologie et Herpetologie 23 (3/4): 91-92.

Triques, M.L. 1997. Stegostenopos cryptogenes, new genus and species of Hypopomidae electrogenic neotropical fish from the Rio Negro system, Brazil (Actinopterygii: Ostariophysi: Gymnotiformes). Révue Française d'Aquariologie et Herpetologie 24 (1/2): 31-36.

Triques, M.L. 1998. Tembeassu marauna, new genus and species of electrogenic neotropical fishes (Ostariophysi: Gymnotiformes: Apteronotidae) Révue Française d'Aquariologie et Herpetologie 25 (1/2): 5-10.

Triques, M.L. 1999. Three new species of Rhamphichthys Müller
\& Troschell, 1846 (Ostariophysi: Gymnotiformes: Rhamphichthyidae). Révue française d'Aquariologie et Herpetologie 26 (1/2): 1-6.

Triques, M.L. 2005a. Novas sinapomorfias para Rhamphichthys Muller \& Troschel, 1848 (Teleostei: Rhamphichthyidae). Lundiana 6 (1): 35-39.

Triques, M.L. 2005b. Análise cladística de caracteres de anatomia externa e esquelética de Apteronotidae (Teleostei: Gymnotiformes). Lundiana 6 (2): 121-149.

Triques, M.L. \& D.K. Khamis. 2003. Brachyhypopomus jureiae, a new species of freshwater neotropical electric fish (Teleostei: Gymnotiformes: Hypopomidae) from a coastal stream of Southeastern Brazil. Lundiana 4 (1): 61-64.

Watrous, L.E. \& Q.D. WheELER. 1981. The out-group comparison method of character analysis. Systematic Zoology 30 (11): $1-11$.

WeitZMAn, S.H. 1974. Osteology and evolutionary relationships of the Sternopytychidae, with a new classification of stomiatoid families. Bulletin of the American Museum of Natural History 153 (3): 327-478.

Wiley, E.O. 1981. Phylogenetics: The Theory and Practice of Phylogenetic Systematics. New York, John Wiley \& Sons. XVI+439p.

Recebido em 18.XII.2006; aceito em 20.VIII.2007. 